helical bundles that form between transport vesicles and acceptor membranes and are essential for membrane fusion (reviewed in ref. 5). When it binds the SNARE complex, Snap recruits NSF, an ATPase that catalyzes the disruption of the complex. This frees individual SNARE proteins for another round of complex formation and membrane fusion.

$\alpha$ Snap is an essential protein whose levels must be carefully titrated. Chae et al. report that a null mutation in Napa is lethal in mice, and hypomorphic alleles in Drosophila melanogaster lead to reduced neurotransmission (M. Babcock, G.T. Macleod, J. Leither \& L. Pallanck, personal communication). Overexpression of the protein blocks fusion of yeast vacuoles ${ }^{6}$ and disrupts synapse formation and other aspects of development in D. melanogaster (M. Babcock, G.T. Macleod, J. Leither \& L. Pallanck, personal communication).

The hyh mutation does not affect the gross structure of $\alpha$ Snap or its ability to bind SNARE complexes or to recruit and stimulate NSF-catalyzed disassembly of SNARE complexes. Thus, the mutant phenotype seems to be due to a simple reduction in $\alpha$ Snap levels. This suggests that $\alpha$ Snap has a dose-dependent action during ventricular zone proliferation that is essential to producing neural progenitors.

\section{The importance of being apical}

In early cortical development, two main types of cell division are observed ${ }^{7}$ : symmetric and asymmetric. In symmetric cell divisions both daughters inherit the same complement of membrane and cytoplasm and give rise to two new progenitors. In asymmetric cell divisions one daughter inherits the apical half of the mother cell and remains a progenitor whereas the other daughter inherits the basolateral half and becomes a neuron. The differential partitioning of membrane domains and soluble factors in asymmetric cell divisions is proposed to contribute to differences in cell fate (Fig. 1).

Chae et al. report that in hyh mutants the apical localization of several soluble proteins implicated in cell fate is disrupted and that localization of VAMP7, a vesicular SNARE that participates in apical targeting ${ }^{8}$, is less polarized than in wild-type mice. This suggests that the phenotype could result from a reduction in SNARE-mediated delivery of scaffolding proteins to the apical surface. It also supports a model in which becoming a progenitor is the non-default fate, one that must be actively maintained with sufficient apical signaling machinery. Thus, the decision that must be induced is to prolong proliferation, rather than to initiate differentiation.

Identifying the genes responsible for naturally occurring mutations often leads to sur- prising revelations about cellular processes and to new questions about old players. The seemingly pronounced sensitivity of cortical neurogenesis to levels of $\alpha$ Snap suggests that the shared machinery of membrane trafficking may not function equally throughout the cell. Future studies comparing apical to basolateral sorting in hyh mutant progenitor cells, examining the organization of other polarized tissues and determining whether other Snap isoforms can rescue the phenotype will expand our understanding of snap function. Analyses of other cell fate determinants in hyh mutants will help identify the role these molecules have in the decisions that produce complex cellular patterns. The findings certainly highlight the importance of distinct plasma membrane domains in polarized cells and suggest that they could be orchestrating cell fate determination during neurogenesis.

1. McConnell, S.K. Neuron 15, 761-768 (1995).

2. Caviness, V.S. Jr., Takahashi, T. \& Nowakowski, R.S. Trends Neurosci. 18, 379-383 (1995).

3. Chae, T.H., Kim, S., Marz, K.E., Hanson, P.I. \& Walsh, C.A. Nat. Genet. 36, 264-270 (2004).

4 Hong, H.K., Chakravarti, A. \& Takahashi, J.S. Proc. Natl. Acad. Sci. USA 101, 1748-1753 (2004).

5. Hay, J.C. Exp. Cell Res. 271, 10-21 (2001).

6. Wang, L., Ungermann, C. \& Wickner, W. J. Biol. Chem 275, 22862-22867 (2000).

7. Chenn, A. \& McConnell, S.K. Cell 82, 631-641 (1995).

8. Lafont, F. et al. Proc. Natl. Acad. Sci. USA 96, 3734-3738 (1999)

\title{
Sox3 and sexual dysfunction: it's in the head
}

\author{
Sally A Camper
}

\section{Humans with mutations in SOX3 have panhypopituitarism, but the developmental mechanisms underlying this defect are unknown. Conditional disruption of Sox3 in mice now suggests that anterior pituitary development depends on Sox3 expression in the overlying neural ectoderm, which establishes midline structures and regulates production of inductive BMP and FGF signals.}

\begin{abstract}
Thirteen years ago, Lovell-Badge and colleagues proved that Sry is the mammalian testis-determining gene by showing that Sry was sufficient to convert XX mouse embryos into phenotypic males ${ }^{1}$. Sry is the founding member of a large family of related genes called SOX genes (for SRY-related HMGbox). The SOX DNA-binding domain, called HMG for high mobility group, is the most highly conserved region of these proteins.
\end{abstract}

Sally A. Camper is in the Department of Human Genetics, University of Michigan Medical School, Ann Arbor, Michigan 48109-0638, USA. e-mail: scamper@umich.edu
Do other SOX genes also function in sex determination? Haploinsufficiency with respect to SOX9 causes campomelic dysplasia, a syndrome characterized by skeletal abnormalities and ambiguous genitalia or sex reversal ${ }^{2,3}$. Mice with mutations in $O d s$ and gain of Sox9 function also have sex reversal, identifying the importance of Sox9 gene dosage ${ }^{4}$. Another member of the SOX family, Sox 3 , is closely related to $S r y$, but its role in sex determination is controversial ${ }^{5,6}$. On page 247 Rizzoti et al. report that Sox3, unlike Sry or Sox9, is dispensable for directing the indifferent gonad to develop into an ovary or testis on a mixed genetic background? ${ }^{7}$.
Instead, Sox3 seems to affect gonadal function indirectly through its roles in brain development and hypothalamic induction of anterior pituitary development.

\section{Sox on the brain}

The phenotype of Sox3 mutant mice is variable and complex, with abnormalities throughout the hypothalamic-pituitarygonadal axis (Fig. 1). Severely affected mice have craniofacial abnormalities, defects in the brain midline, profound growth insufficiency, male hypogonadism and lethality, whereas less affected mice are viable and fertile. The expression pattern of Sox3 provided 

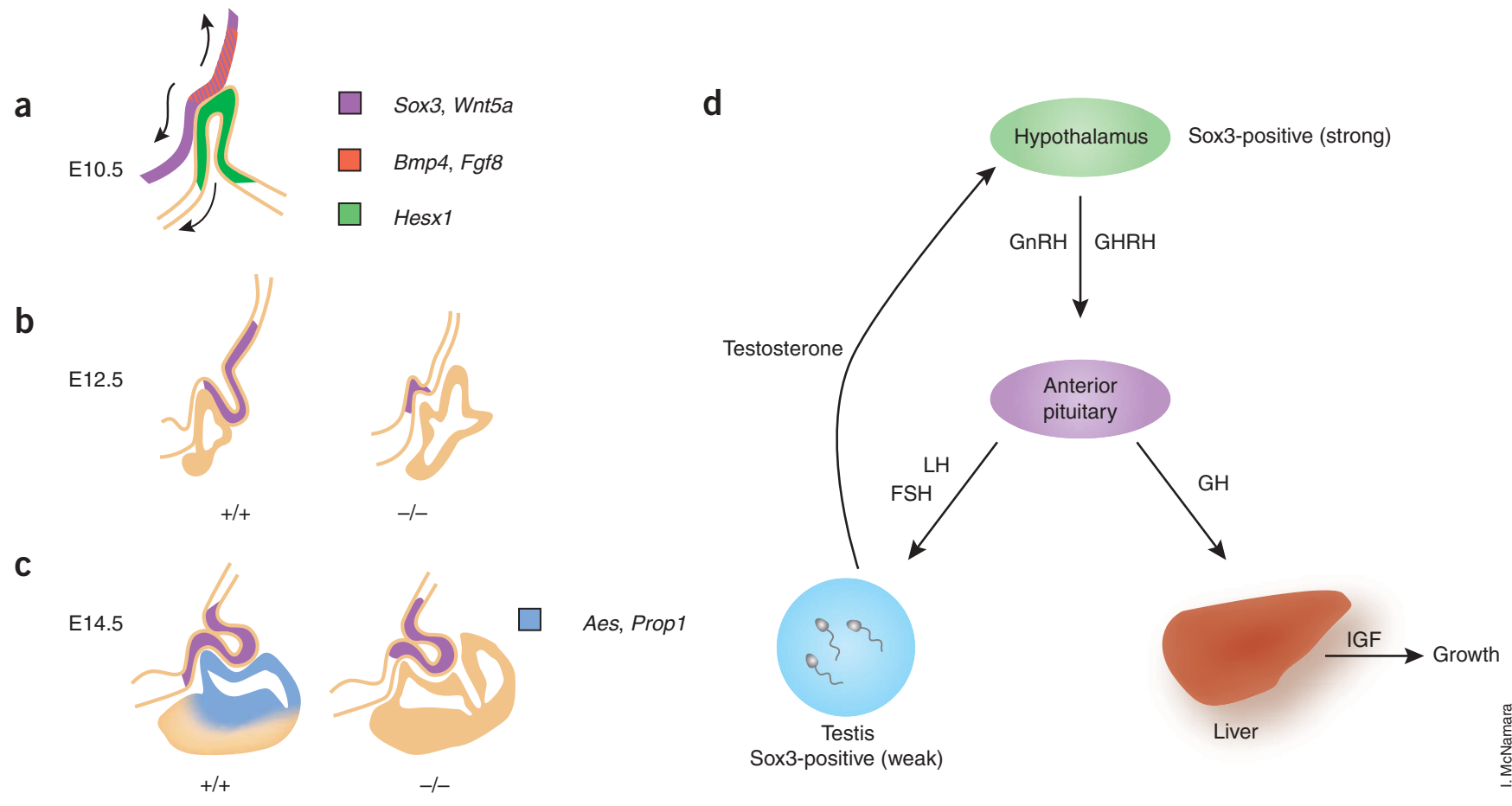

Figure 1 Multiple causes of anterior pituitary dysmorphology and hormone deficiency. (a) Sox3 and Wnt5a are expressed in the ventral diencephalon. Sox3 deficiency results in spreading of Bmp4 and Fgf8 expression within the diencephalon and Hesx 1 expression within the pouch (E10.5 arrows). (b) Anterior pituitary dysmorphology caused by Sox3, Wnt5a, or Hesx1 deficiency is evident at E12.5. The lumen of Rathke's pouch is branched in mutants (sagittal view, right). The area of the brain just above the pouch is also dysmorphic in Sox3 and Hesx 1 mutants, suggesting midline defects. (c) Defects in Aes or Prop1, both intrinsic to the anterior pituitary, result in pouch dysmorphology later in development (E14.5). (d) Anterior pituitary function depends on factors such as $\mathrm{GnRH}$ (gonadotropin releasing hormone) and GHRH (growth hormone releasing hormone) released from the hypothalamus into the hypophyseal portal system. In turn, these hormones cause release of luteinizing hormone (LH), follicle stimulating hormone (FSH), and growth hormone (GH) from the pituitary. LH and FSH stimulate testosterone production and spermatogenesis in the testis, and testosterone feeds back to the hypothalamus to maintain function of the hypothalamic-pituitary-gonadal axis. Sox3 is expressed at high levels in the median eminence of the hypothalamus and within selected cells of the testis, but not in the anterior pituitary gland. Thus, the pituitary hypoplasia, infertility and growth insufficiency of Sox3 mutants likely result from primary defects in the ventral hypothalamus and secondary defects in the pituitary gland that ultimately affect target organs.

a clue for dissecting primary from secondary effects of Sox3 deficiency. Sox3 is not expressed in Rathke's pouch, the structure that gives rise to the anterior and intermediate lobes of the pituitary, yet the anterior lobes of Sox3 mutant mice are hypoplastic and the lumen separating the intermediate and anterior lobes is branched and dysmorphic (Fig. 1). In contrast, Sox3 is highly expressed in the ventral hypothalamus and throughout the infundibulum, a neural ectoderm-derived structure that develops into the posterior lobe of the pituitary and pituitary stalk.

Classic tissue recombination experiments in chick and frog have shown that contact between the infundibulum and Rathke's pouch is required for differentiation of the hormone-producing cells of the anterior pituitary lobe, but little is known about the molecular nature of this inductive process. The transcription factor Nkx2.1 and the signaling proteins FGF8 and BMP4 are known to be important for cell survival and cell specification, respectively ${ }^{8,9}$. Rizzoti et al. now show that Sox3 deficiency results in an expansion of FGF8 and BMP4 expression and reduced proliferation in the infundibulum, suggesting that Sox3 normally patterns pouch development by restricting signaling domains and stimulating growth of the infundibulum.

The bifurcated Rathke's pouch in Sox3 mutants is reminiscent of pituitary gland abnormalities reported in other mouse mutants (Fig. 1). For example, absence of the transcription factor Hesx 1 results in dysmorphology of the pituitary gland and ventral hypothalamus similar to that seen in Sox 3 mutants ${ }^{10}$, supporting the idea suggested by Rizzoti et al. that pituitary defects in Hesx 1 -deficient mice are secondary to the midline defects and not intrinsic to the gland itself. Wnt5a, a secreted signaling molecule, is also expressed in the infundibulum at approximately the same time as Sox3, and mice lacking either gene have nearly identical anterior pituitary dysmorphology ${ }^{11}$. Wnt5a and Sox3 might therefore exert similar effects on BMP or FGF signaling, perhaps by restricting expression or antagonizing the effects of these signaling molecules. Mice lacking the transcription factors Pitx2, Tcf7l2 (TCF4) or Aes (groucho-related gene 5) also have defects in pituitary growth and shape ${ }^{12,13}$. Taken together, these data suggest that the mechanism for induction of anterior pituitary differentiation and regulation of organ shape is complex and requires inputs from multiple genes.

\section{Green clusters}

Weiss et al. recently reported the effects of a targeted deletion of Sox3, but their study focused on testicular and ovarian phenotypes and did not examine brain or pituitary development in detail ${ }^{14}$. Although the Sox3 knockouts are similar in design, Rizzoti et al. inserted a green fluorescent protein marker 
into the Sox3 locus that allowed for detection of cells that normally express Sox3. The infundibulum of Sox3 heterozygous females was preferentially composed of cells with an inactivated wild-type $\mathrm{X}$ chromosome, which explains the pituitary dysmorphology seen in most heterozygous females. The molecular mechanism underlying this unexpected enrichment of Sox3 mutant cells in the infundibulum of female heterozygotes is an interesting avenue for future studies.

The expression of Sox3 in the urogenital ridge and adult testis leaves open the possibility that the loss of male germ cells and testis necrosis in older mice could be attributed to testicular expression of Sox3. But the pituitary hypoplasia and hormonal abnormalities clearly suggest that the brain expression of Sox3 is important for maintaining function of the hypothalamic-pituitarygonadal axis. Clarification of this issue will require tissue-specific Cre-mediated deletion of Sox3 in both tissues and careful comparison of the resulting phenotypes.

Although the full contribution of genetic background to the variability of the Sox3 mutant phenotype has yet to be explored, the observed variability among Sox3 knockout mice suggests that a range of phenotypes might be associated with SOX 3 abnormalities in humans. As Rizzoti et al. point out, there is a good chance that mutations in SOX3 may account for some fraction of sporadic hypopituitarism or growth insufficiency in males. Notably, individuals with duplications of Xp26 encompassing the SOX3 gene have mental retardation and hypopituitarism, suggesting that brain and pituitary development might also be sensitive to SOX3 dosage ${ }^{15}$. Analysis of Sox3 gain-of-function mice and analysis of additional humans with SOX3 alterations will be particularly informative in unraveling the developmental functions of this gene and its contribution to pituitary dysfunction in humans.

1. Koopman, P., Gubbay, J., Vivian, N., Goodfellow, P. \& Lovell-Badge, R. Nature 351, 117-121 (1991).

2. Foster, J.W. et al. Nature 1372, 525-530 (994).

3. Wagner, T. et al. Cell 79, 1111-1120 (1994).

4. Bishop, C.E. et al. Nat. Genet. 26, 490-494 (2000).

5. Stevanovic, M., Lovell-Badge, R., Collignon, J. \& Goodfellow, P.N. Hum. Mol. Genet. 2, 2013-2018 (1993).

6. Graves, J.A. Bioessays 20, 264-269 (1998)

7. Rizotti, K. et al. Nat. Genet. 36, 247-255 (2004).

8. Takuma, N. et al. Development 125, 4835-4840 (1998).

9. Ericson, J., Norlin, S., Jessell, T.M. \& Edlund, T. Development 125, 1005-1015 (1998).

10. Dattani, M.T. et al. Nat. Genet. 19, 125-133 (1998).

11. Cha, K.B. et al. Mech. Dev. advance online publication, 9 January 2004 (doi:10.1016/j.mod.2003. 12.002).

12. Brinkmeier, M.L. et al. Mol. Endocrinol. 17 , 2152-2161 (2003)

13. Kioussi, C. et al. Cell 111, 673-685 (2002).

14. Weiss, J. et al. Mol. Cell. Biol. 23, 8084-8091 (2003).

15. Solomon, N.M. et al. Genomics 79, 553-559 (2002) 